\title{
DESIGN AND DEVELOPMENT OF 4-BYTE SRAM ARCHITECTURE
}

\author{
Datti Atchutarao \\ PG scholar, Department of Electronics and Communication Engineering, \\ GMR Institute of Technology, Rajam, Andhra Pradesh, India \\ V. Kannan \\ Professor, Department of Electronics and Communication Engineering, \\ GMR Institute of Technology, Rajam, Andhra Pradesh, India
}

\begin{abstract}
In VLSI, there is integration of hundreds and thousands of transistors to form a chip (or) microchip. SRAM is utilized for computer cache memory and a chunk of random access memory digital to analog converter on a video card. SRAM keeps data constant, without the need of memory module to be refreshed periodically. SRAM takes main role in very large scale integrated circuit (VLSI) due to its storage capacity and small access time. In this work the simulation study of different SRAM cells 4+2T SRAM, 4TSRAM, 5T SRAM and 6T SRAM are done. Also a 4byte SRAM architecture with row decoder, column decoder, SRAM cell, pre-charge circuit, sense amplifier and write driver circuit are simulated using EDA tool in 45nm technology. The average power delay and area various SRAMs are calculated and compared. SRAM$5 T$ is showing better results for Average power consumption and delay while SRAM$4 T$ is better in terms area.
\end{abstract}

Key words: SRAM, Decoder, Pre-charge circuit, Sense amplifier, Write driver circuit, Tanner EDA, VLSI.

Cite this Article: Datti Atchutarao and V. Kannan, Design and Development of 4-Byte SRAM Architecture. International Journal of Electrical Engineering and Technology, 11(5), 2020, pp. 31-42.

http://iaeme.com/Home/issue/IJEET?Volume=11\&Issue $=5$

\section{INTRODUCTION}

Very large scale integration is the pursuit of synthesize a thousands and million of transistors within a isolated chip. Very large scale integration is the pursuit of synthesize a thousands and million of transistors within an isolated chip. So many researchers are working in the field of VLSI and Nano Technology, in that Static random access memory (SRAM) is a leading chunk in most of VLSI chips [1-3]. SRAM has nether access time disparity to DRAM. The conventional von-Neumann $(\mathrm{CVN})$ architecture is having three major steps memory, registers, arithmetic logical unit (ALU). 1) Memory is for storing purpose, 2) Registers to 
grasp data, 3) ALU to execute arithmetic and logical operations. During memory computing (IMC), it operates various rows simultaneously and carry-out logical functions from bit line (BL). Both latency and energy are governed by two memory accesses and data movement. As a result, IMC can precipitate the energy costs integrated with memory access and data moment. While approaching IMC with different types of SRAMs, 4+2T SRAM is suitable for easy to memory access and data movement [4]. The proposed 4+2T SRAM cell is used with n-well write operations. SRAM can be configured as Binary content addressable memory (BCAM) and Ternary content addressable memory (TCAM). There are many recent proposals for Near data processing (NDP) architectures focusing essentially on In-memory analytics [5]. NDP systems eliminate the energy over-heads of moving data over long board traces. The NDP runtime exposes a set of API functions that initialize and execute software on the NDP hardware [6]. It co-ordinates with the OS running in host cores for file $\mathrm{I} / \mathrm{O}$ and exception handling. Figure 1. Shows the data flow required for reading two words to perform logic operation in CVN architecture and IMC system. Figure 2. Shows the contrast between CVN and IMC in terms of (a) Latency and (b) energy for a two-word Boolean operation.
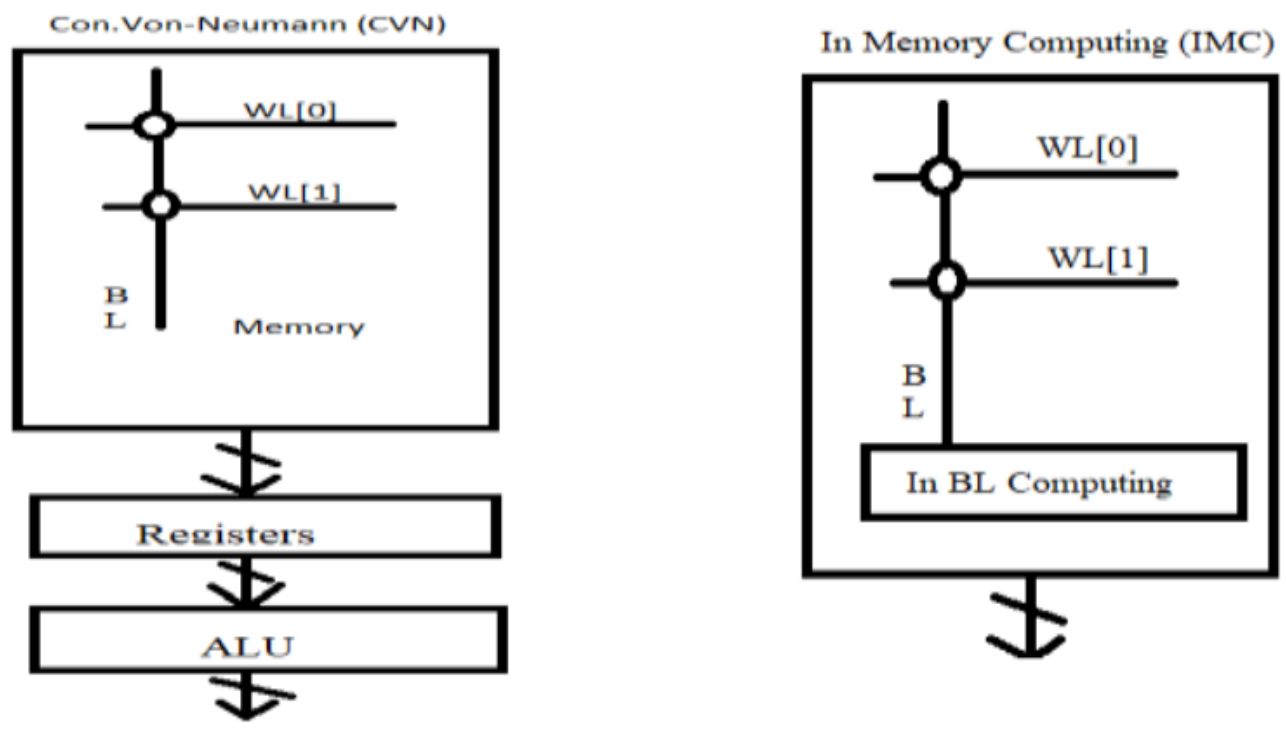

Figure 1 Data flow required for reading two words to perform logic operation In CVN architecture and IMC system

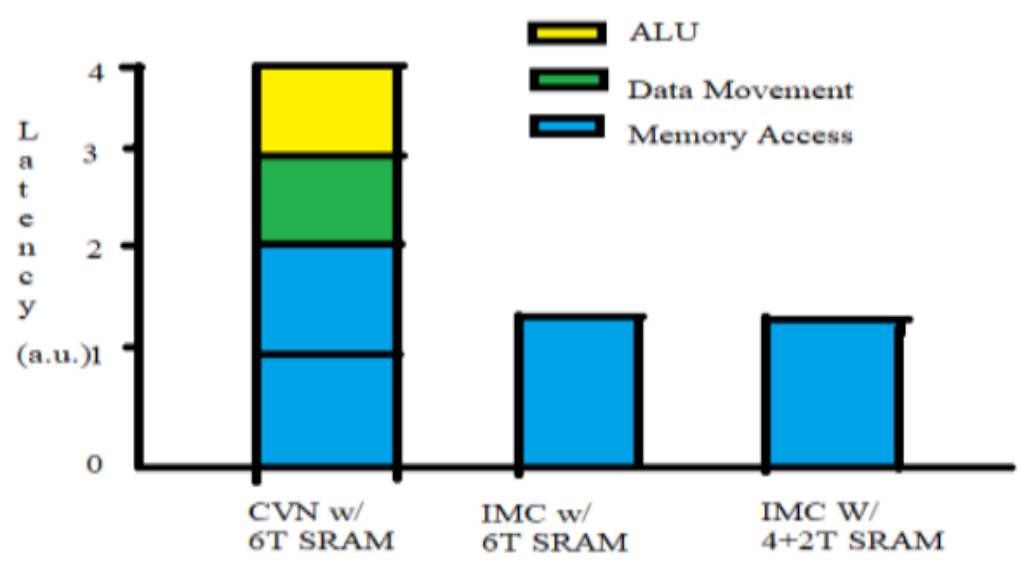

(a) 


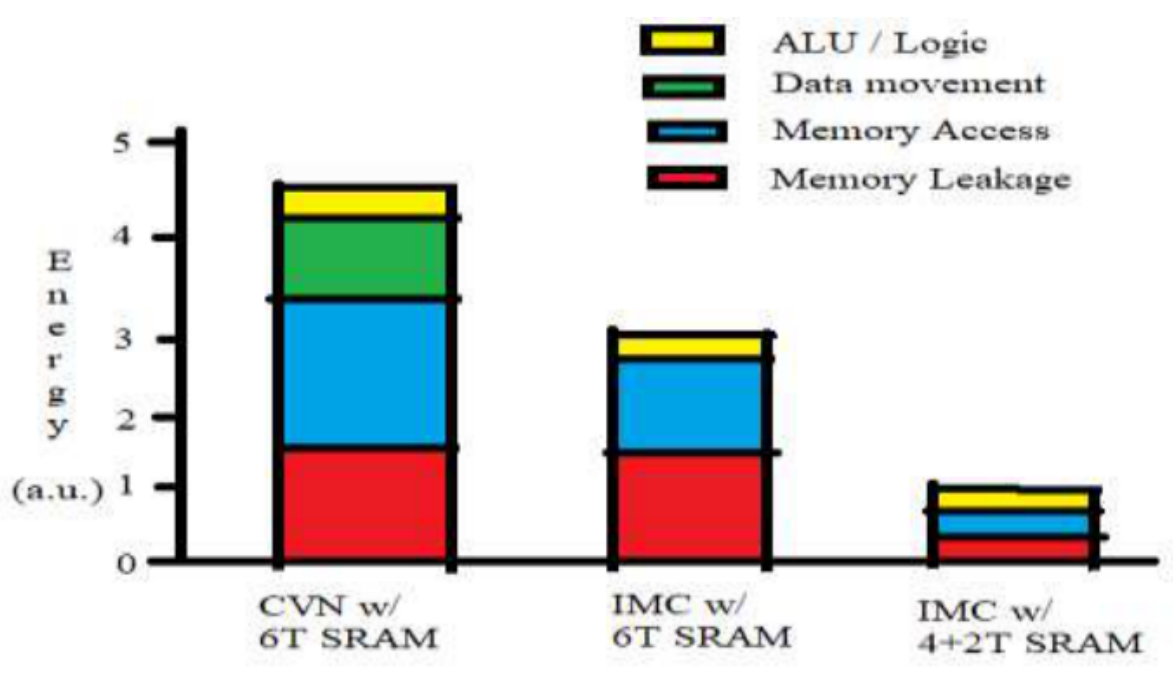

(b)

Figure 2 Contrast between CVN and IMC in terms of (a) Latency and (b) energy for a two-word Boolean operation.

\section{TYPES OF SRAM CELL}

The SRAM Cell is a type of flipflop circuit, usually implemented using MOSFET (Metal oxide semiconductor field effect transistor). The various categories of SRAM Cells considered in this paper are 4+2T SRAM, 4TSRAM, 5T SRAM and 6T SRAM.

\subsection{T SRAM Cell}

All survive SRAM Cells can be split into two categories like 6T SRAM Cell and 4T SRAM Cell having charge element. The 4T SRAM Cell have influence the stand alone SRAM market since they have much less area than 6T SRAM Cell. The schematic of 4T SRAM Cell is illustrated in Fig. 3.

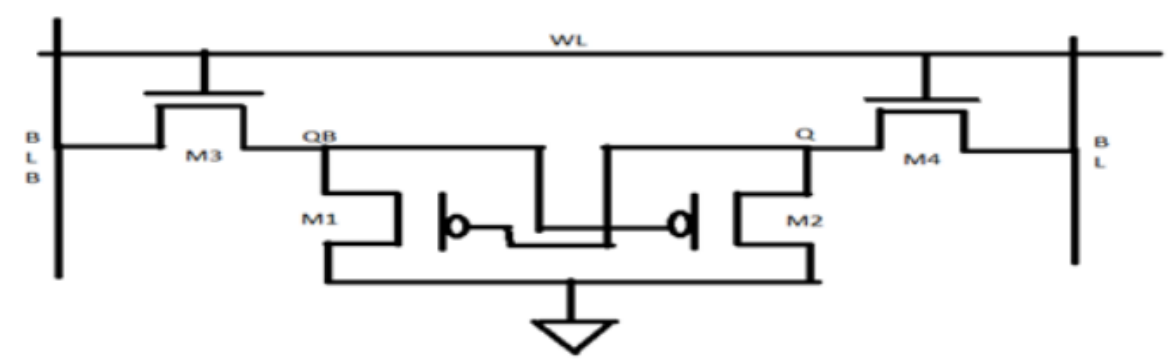

Figure 3 SRAM $4 \mathrm{~T}$

\subsection{4+2T SRAM Cell}

A 4+2T SRAM Cell is advanced that offers searching ang logic functions. This cell uses Nwell as the word line (WL) and terminates the access transistors decoupled read paths enabled reliable multiword activation for memory Boolean logic function. The schematic of $4+2 \mathrm{~T}$ SRAM Cell is illustrated in Fig. 4. 


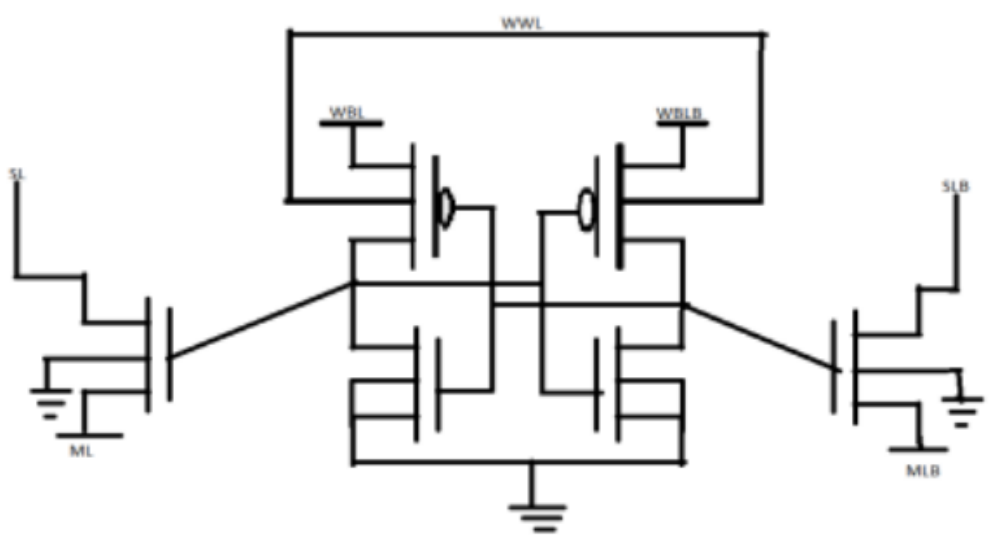

Figure 4 SRAM $4+2 \mathrm{~T}$

\subsection{T SRAM Cell}

This SRAM Cell looks distinguishable like a conventional 6T SRAM Cell, but the only variability is missing of one access transistor. Read and write access are distinguishable to 6T SRAM Cell except they have single ended through the lone access transistor. With all these write assist techniques the overall area saving of the 5T SRAM macro is 5\% compared to $6 \mathrm{~T}$ SRAM [7]. The schematic of 4bit cell is illustrated in Fig. 5.

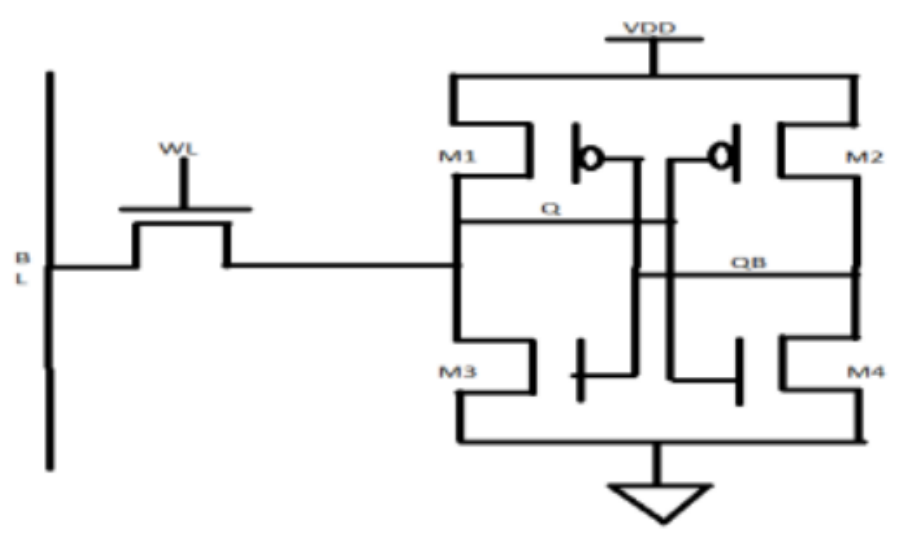

Figure 5 SRAM 5T

\subsection{T SRAM Cell}

The 6T SRAM Cell can be developed by using two PMOS transistors and four NMOS transistors. A typical 6T SRAM consists of two access transistors and two cross coupled inverters. Both inverters are connected back to back with each other. The bit-cell array is identical to a standard 6T SRAM, including sizing. We note that in the classify mode the cells face the potential for a new upset condition [8]. The schematic of 6T SRAM Cell is illustrated in Fig. 6. 


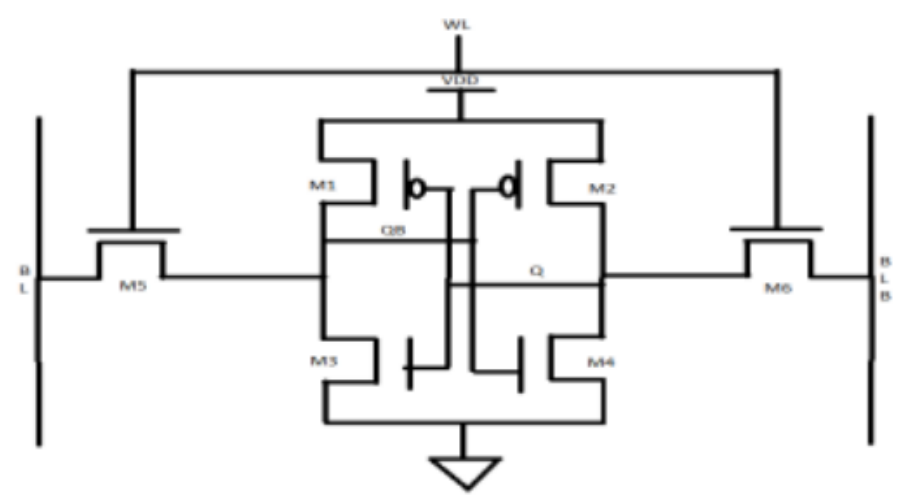

Figure 6 SRAM 6T

\section{CAM CONFIGURATIONS}

Content addressable memory (CAM) is one kind of special memory. It will search entire memory for input data. While finding data it will matching words with address locations. A redesign CAM circuit that enhances array density by as much as $4 \mathrm{x}$ based on traditional pushrule 6T SRAM bit cell is suggested [9]. Data are retrieved by contents rather than physical locations is an exceptional type of solid-state memory called as Content Addressable Memory (CAM) [10]. An exact match is searched by a Binary content addressable memory (BCAM). Whereas a Ternary content addressable memory (TCAM) explore for a restricted matching with "don't care" bits in memory.

\subsection{BCAM Mode}

Binary content addressable memory (BCAM) reserve 1's and 0's with penetrating data for expect match. while keeping ML0, SLB0, SL1 in higher state and SL0, SLB1 in lower state the output of first row-wise sense amplifier is high state. With one side attached to 'Vref' to assess results. If ML1 in nether state the output of second row-wise sense amplifier is nether state. depicted in below figure.

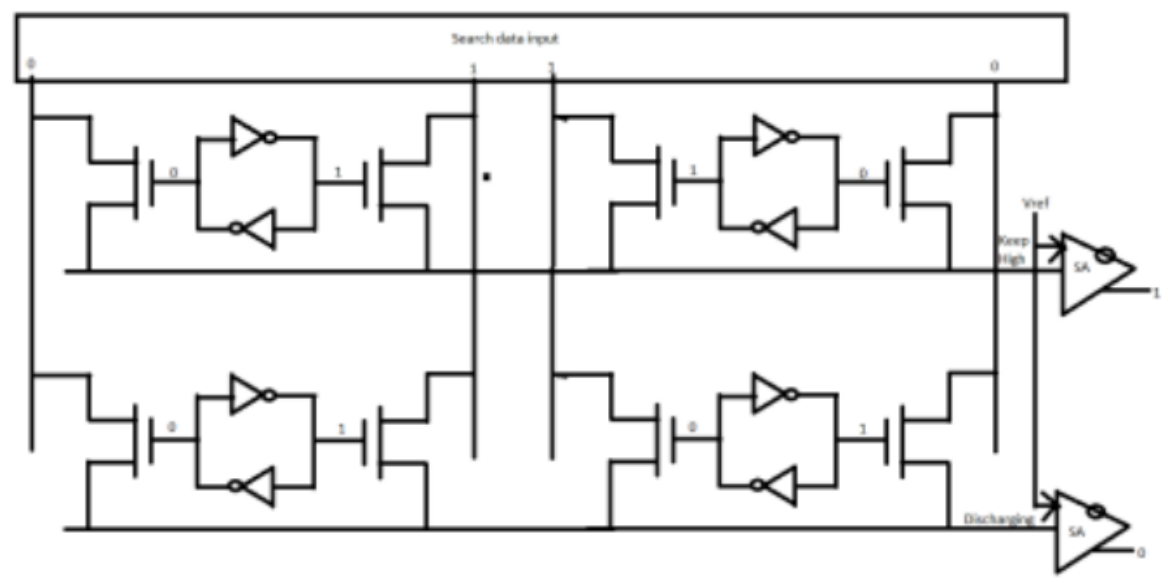

Figure 7 BCAM

\subsection{TCAM Mode}

Ternary content addressable memory (TCAM) reserve 0's, 1's and don't cares with penetrating data for restricted matching. While keeping ML0, SLB0, SL1 in elevated state and SL0, SLB1 in nether state, the output of first row-wise sense amplifier in elevated state. With 
one side connected to 'Vref' to evaluate results. If ML1 in nether state the output of second row-wise sense amplifier is nether state shown in below figure.

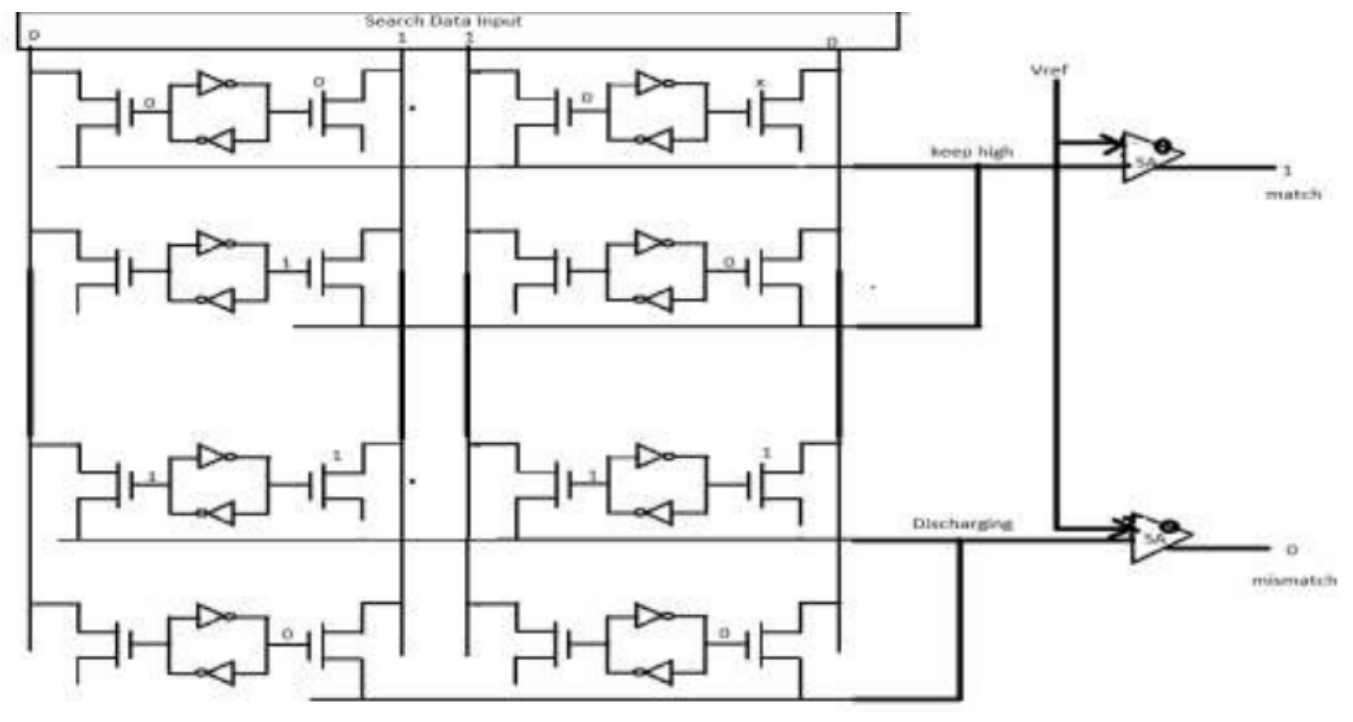

Figure 8 TCAM

\section{DESIGN OF SRAM ARCHITECTURE}

SRAM Architecture is an enfold of SRAM cell, sense amplifier, pre-charge circuit, decoder and write driver circuit. By using SRAM 4T delineate SRAM architecture, because it inhabited low area and delay. The decoder permute the binary directions into unary directions precisely only one word in the array is picked.

The traditional Sparse Distributed memory (SDM) architecture in numerous blocks in sequence to magnify throughput. This is because conventional SRAMs need to employ column multiplexing, where by multiple bit-lines (BLs) share a single sense amplifier (SA) [11]. SRAM is mainly consumed by bit line charge/discharge current. Therefore in sequence to lower the power exhaustion of SRAM, conquer bit line swing is significantly important [12]. while sensitivity to PVT variation is a major challenge for subthreshold logic circuits, some consider it to be prohibitive for memories because of the high design margins required for large scale memories [13]. SRAM cell has different data contrast to the rest of the cells in the same column. The data is arbitrarily stored to SRAM and named as randomized data [14].

\subsection{SRAM Cell}

SRAM cell employ bi-stable latching circuit to gather one bit binary data. The memory cell has two rigid states which are denoted as ' 1 ' and ' 0 '. During write and read operation two additional NMOS transistors control the access to storage cell. The conventional SRAM cell utilize six MOSFET to store data in memory as shown in figure 9. below.

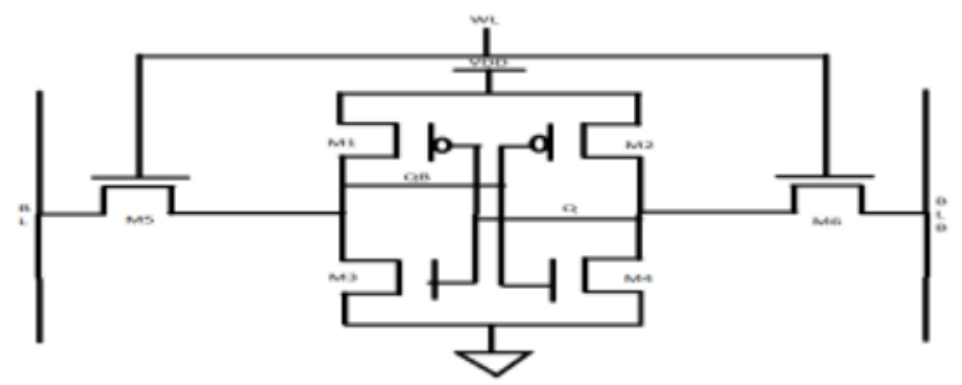

Figure 9 SRAM CELL 


\subsection{Sense Amplifier}

Sense amplifier is the fragment of read circuitry that employ to read the statistics from the SRAM Architecture. Its character is to hearing the low power signals from bit lines. Offsets in sense amplifiers appears as a result of global and local variation in their device. Global disparity cites to die-to-die disparity in devices, and local disparity refers to conflict between devices within the same die situate close to each other [15]. The ability of a sense amplifier to sense small signal input differences is limited by its input referred offset [16]. Differential sense amplifier is depicted in figure 10. below.

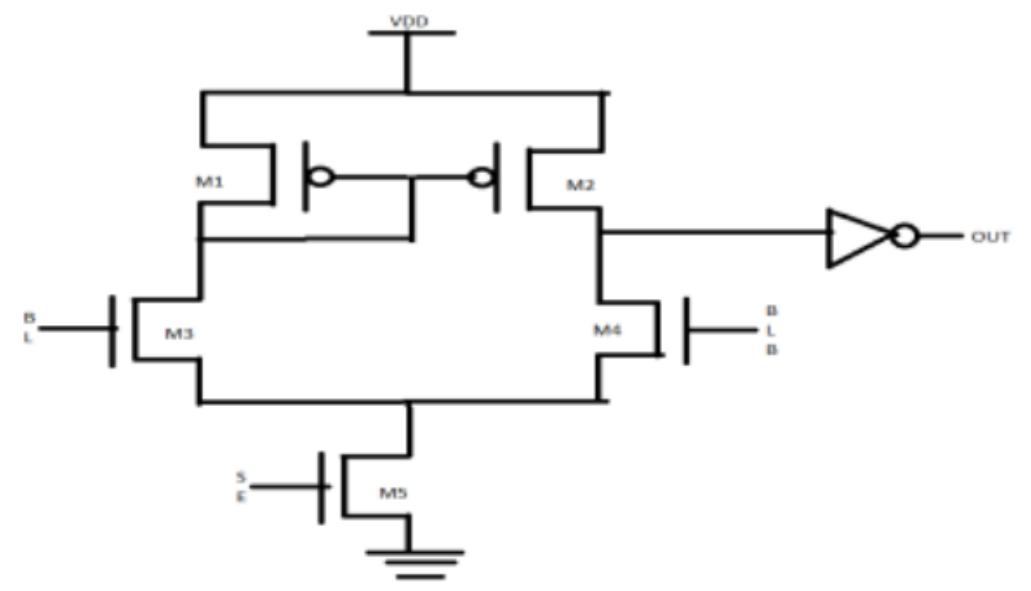

Figure 10 Sense Amplifier

\subsection{Pre-Charge Circuit}

Pre-charge circuit consists of 3 PMOS transistors. Two upper transistors are used for precharge and lower one is used for equalization. Pre-charge circuit is depicted in figure 11. below.

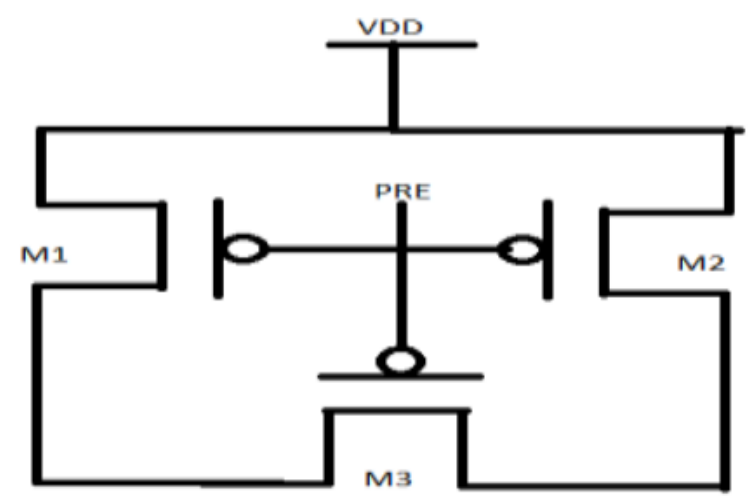

Figure 11 Pre-charge circuit

\subsection{Write Driver Circuit}

Write driver circuit is utilized to emit any one of the bit lines from pre-charge level. It is empowered by a write enable circuitry. In the each column of memory array only one driver circuit is need. 


\subsection{Decoder}

Decoder is utilized to translate the given address and allow the specific row or column of the memory array. Decoder is used to empower word line and write enable of a SRAM array.

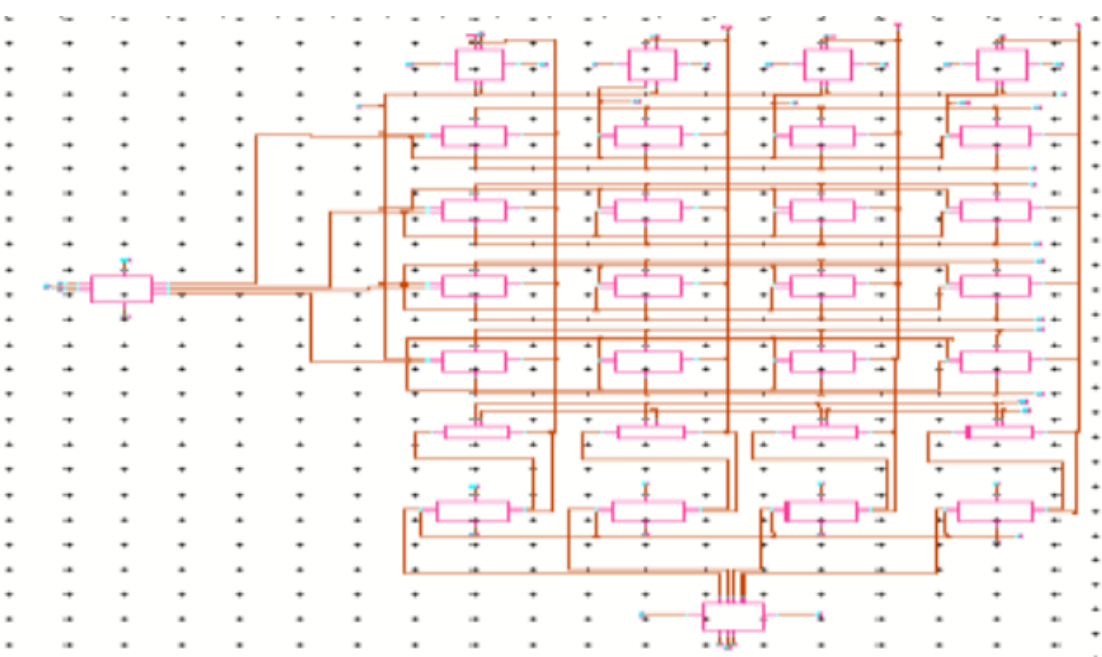

Figure 12 SRAM Architecture

\section{SIMULATION RESULTS}

The low power SRAM Architecture is designed using SRAM cell, Sense amplifier, Write driver circuit, pre-charge circuit and decoder. The absolute work is carried using Tanner EDA $180 \mathrm{~nm}$ technology. The simulation result identify the analysis of different parameters like leakage power and delay. Figure 12. Shows the SRAM architecture. Table 1. Shows the performance comparison of SRAM4T, SRAM4+2T,SRAM 5T AND SRAM 6T.

Table 1 Comparison of power, delay and area of different SRAMs

\begin{tabular}{|c|c|c|c|c|}
\hline & SRAM 4T & SRAM 4+2T & SRAM 5T & SRAM 6T \\
\hline Average Power & $244.2 \mathrm{E}-12$ & $565.7 \mathrm{E}-12$ & $8.487 \mathrm{E}-9$ & $385.1 \mathrm{E}-12$ \\
\hline Delay & $993.5 \mathrm{E}-9$ & $30.04 \mathrm{E}-9$ & $1.018 \mathrm{E}-6$ & $8.650 \mathrm{E}-9$ \\
\hline Area & 2.46490 & 3.705625 & 3.0625 & 3.667255 \\
\hline
\end{tabular}

The average power delay and area various SRAMs are calculated and compared. It is found that SRAM-5T is showing better results for Average power consumption and delay while SRAM-4T is better in terms area.

Figure 13 depicts the simulation of 4T SRAM, which shows timing graph for read and write operation. Normally, stand by mode is not used in empirical applications of any memory devices. So, this work focus on write state and read state alone. 


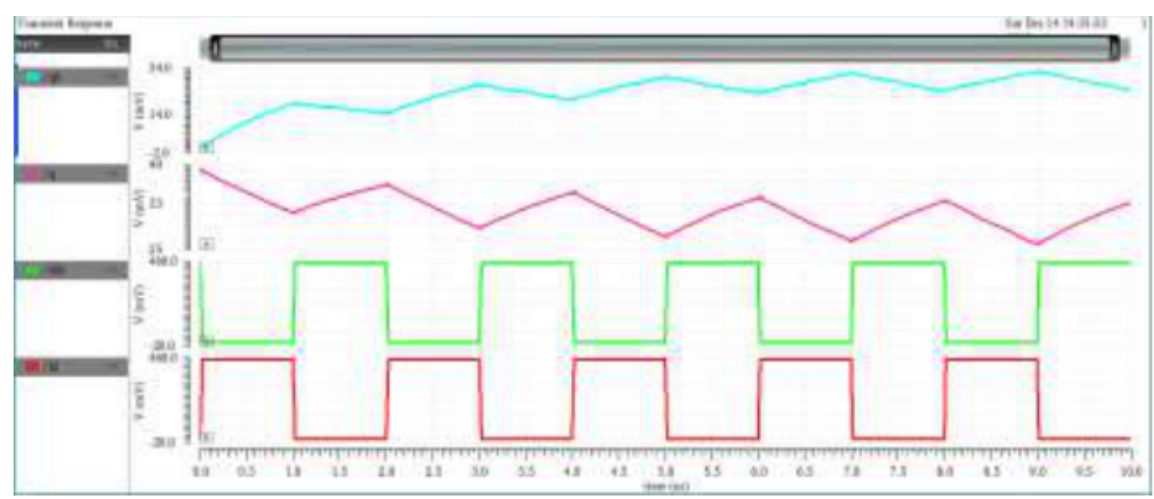

Figure 13 SRAM 4T

Figure 14 depicts the simulation of 4+2T SRAM cell, with its input voltage and output with respect to time. The speed of retaliation is high compared to conventional 6T SRAM cell.

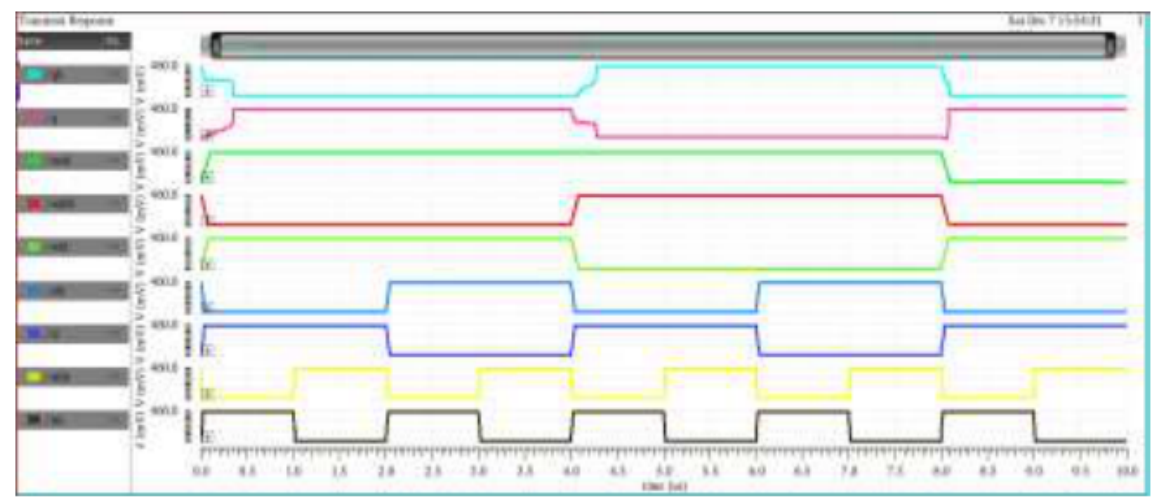

Figure 14 SRAM $4+2 \mathrm{~T}$

Figure 15 depicts the simulation of 5T SRAM cell, with its input voltage and output with respect to time. The speed of reaction is high compared to conventional 4T SRAM cell.

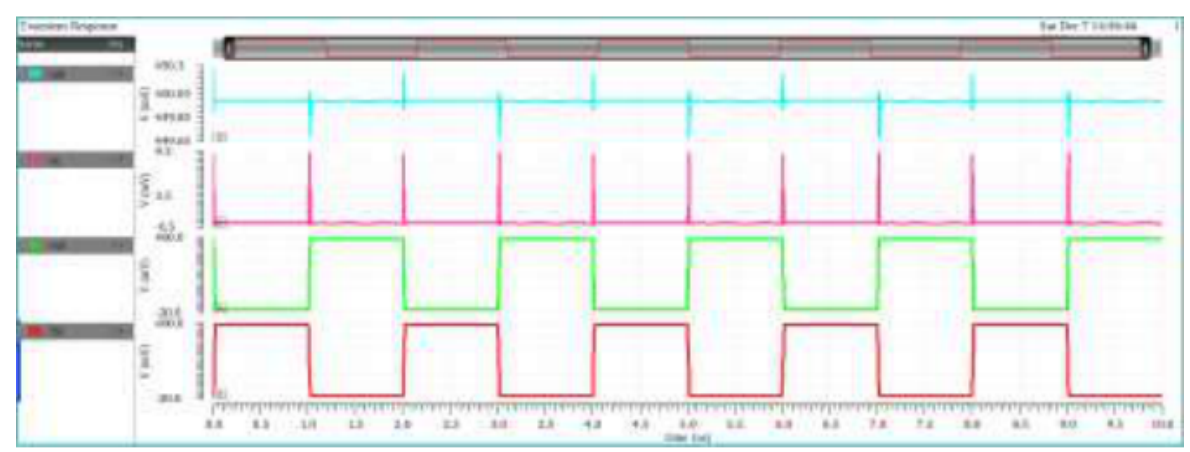

Figure 15 SRAM 5T

Figure 16 depicts the simulation of 6T SRAM cell. It perceive that the response is same but the changes given conventional $6 \mathrm{~T}$ output voltage is due to some ephemeral delay present in the design itself. 


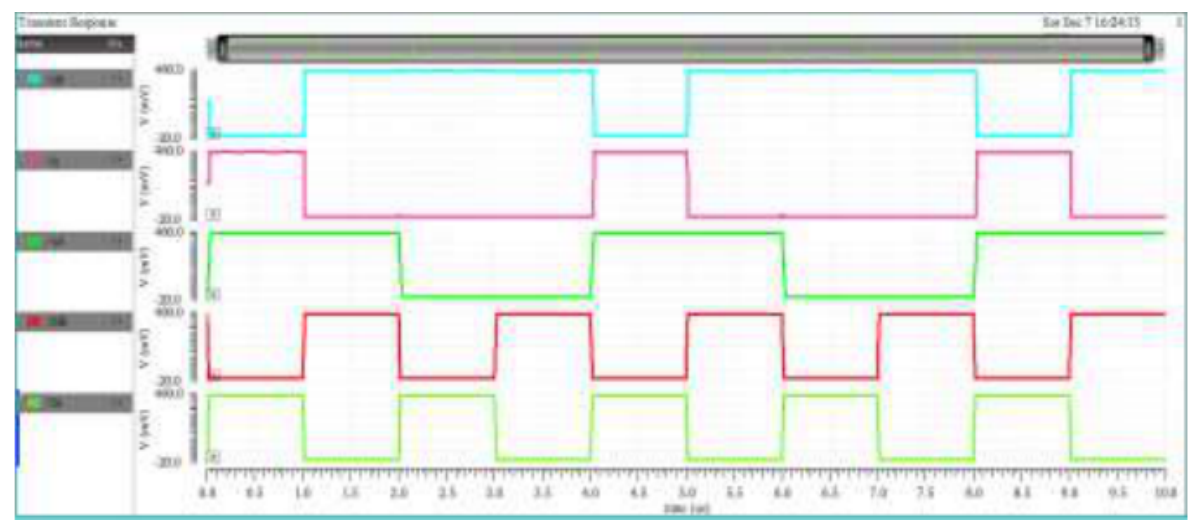

Figure 16 SRAM $6 \mathrm{~T}$

Binary content addressable memory (BCAM) stores zero's and one's with searching data for exact match. while keeping SL0, SL1in higher state and SLB0, SLB1 in lower state the output of first row-wise sense amplifier is in high state. With one side connected to 'Vref' to evaluate results. If ML1 is in lower state the output of second row-wise sense amplifier is in lower state. shown in figure 17. below.

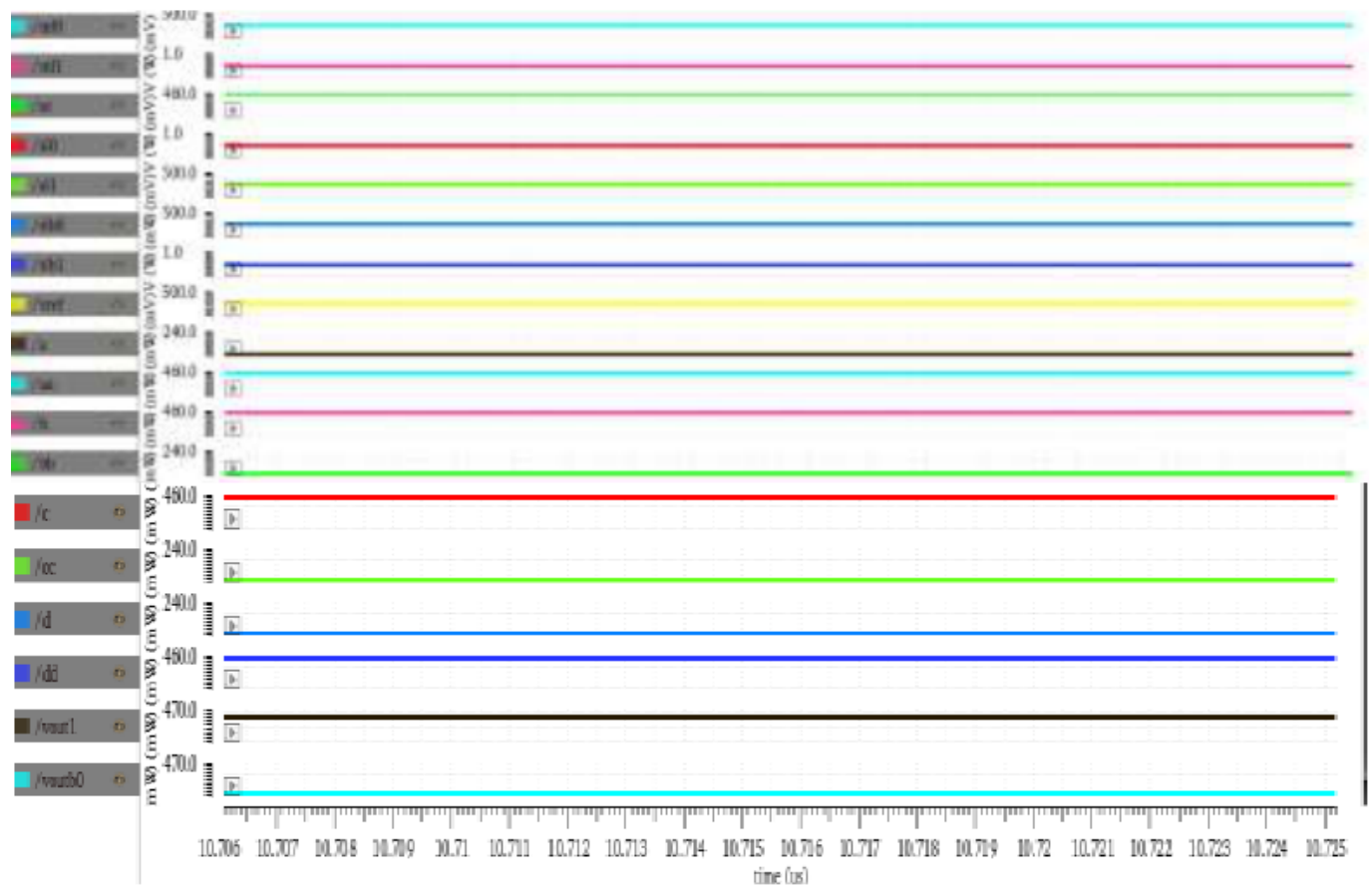

Figure 17 BCAM

Ternary content addressable memory (TCAM) stores zero's, one's and don't cares with searching data for partial matching. While keeping SL0, SL1 in high state and SLB0, SLB1 in low state the output of first row-wise sense amplifier is in high state. With one side connected to 'Vref' to evaluate results. If ML1 in low state the output of second row-wise sense amplifier is low state as shown in figure 18. below. 


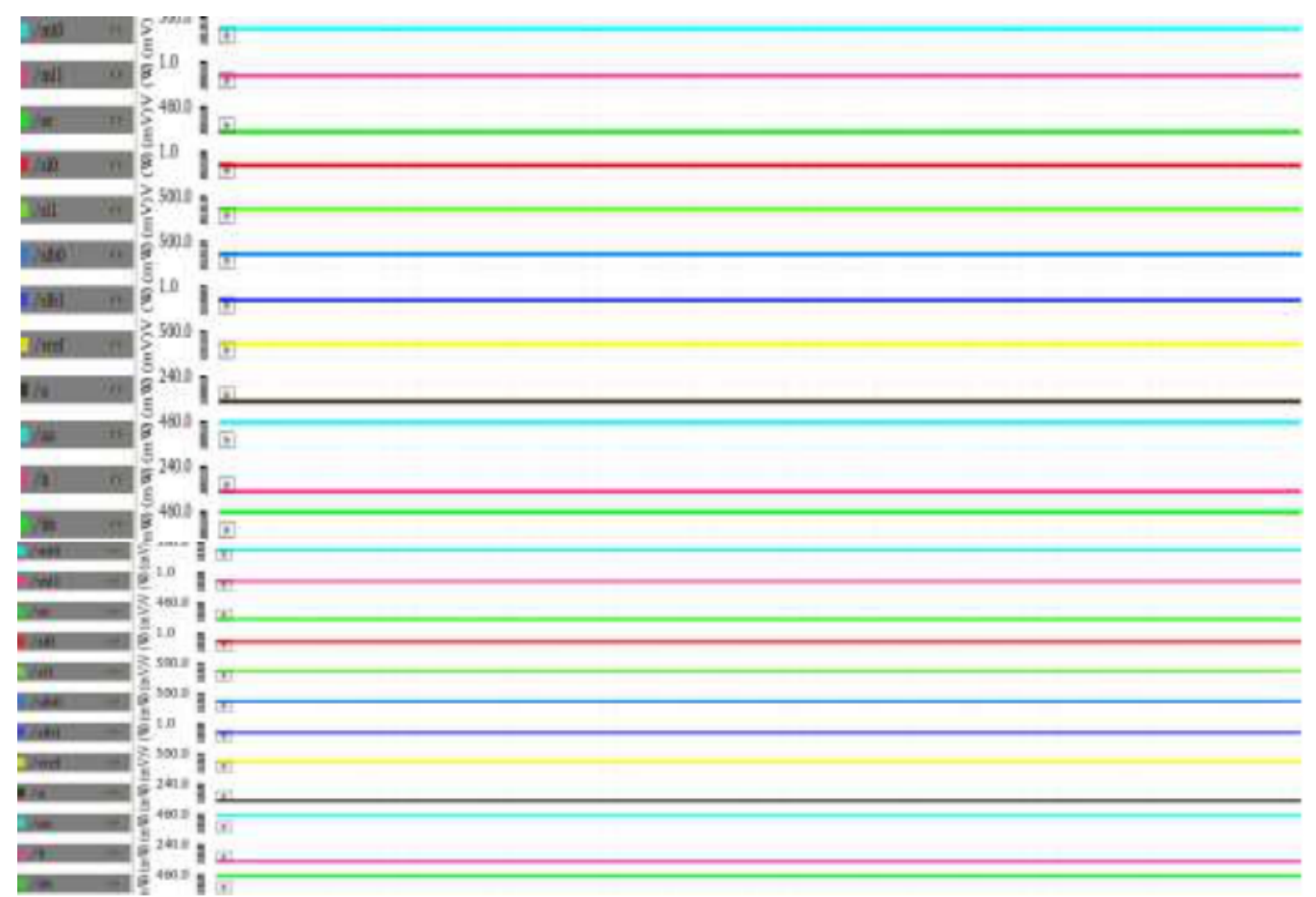

Figure 18 TCAM

During read operation, when both the inputs acquire low, at that time data is being kept at the constant value. When $\mathrm{w}$ goes from high to low and y goes from low to high with sense enable gets on, it confirms the read operation of the memory cell. Figure 19. shows the output timing graph of SRAM architecture.

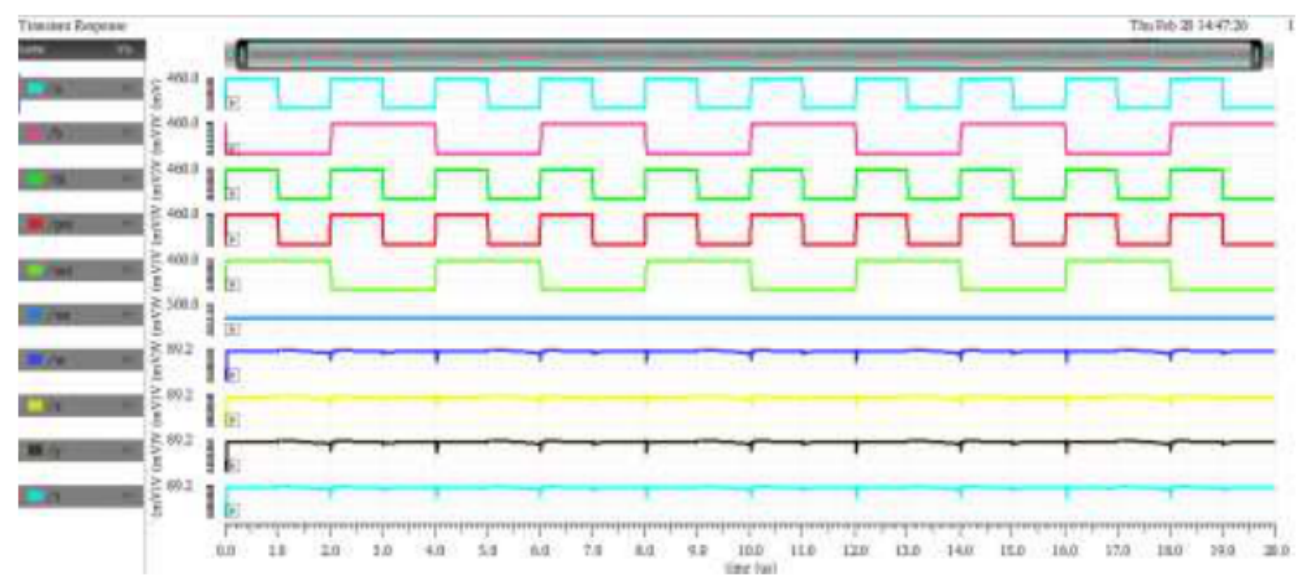

Figure 19 SRAM Architecture

\section{CONCLUSION}

In this work the simulation study of different SRAM cells 4+2T SRAM, 4TSRAM, 5T SRAM and 6T SRAM are done. Also a 4byte SRAM architecture with row decoder, column decoder, SRAM cell, pre-charge circuit, sense amplifier and write driver circuit are simulated using EDA tool in $45 \mathrm{~nm}$ technology. The average power delay and area various SRAMs are calculated and compared. SRAM-5T is showing better results for Average power consumption and delay while SRAM-4T is better in terms area. 


\section{REFERENCES}

[1] S. Pousia and R. Manjith, (2018) "Design of low power high speed SRAM architecture using SK-LCT Technique," in IEEE International conference on Current Trends towards Technologies, Coimbatore.

[2] T Ravi, V Kannan, (2010) Modeling and performance analysis of ballistic carbon nanotube field effect transistor (CNTFET), IEEE, Recent Advances in Space Technology Services and Climate Change 2010 (RSTS \& CC-2010),pp. 285-289

[3] T Ravi, S Ranjith, V Kannan, (2013) A Novel Design of D-Flip Flop Using New RR Fault Tolerant Reversible Logic Gate, International Journal of Emerging Technology and Advanced Engineering (IJETAE), Vol3, issue2

[4] Qing Dong and Supreet Jeloka, (2018) "A 4+2T SRAM for Searching and In-Memory Computing with 0.3-V Vdd min," in IEEE Journal of Solid-State Circuits, vol.53.no.4.

[5] M. GSao and C. Kozyrakis, (2016) "HRL: Efficient and flexible reconfigurable logic for neardata processing," in Proc. IEEE Symp. High Perform.Comput. Archit., Mar. pp. 126-137.

[6] M. Gao, G. Ayers, and C. Kozyrakis, (2017) "Practical near-data processing for in-memory analytics frameworks," in Proc. IEEE Int. Conf. Parallel Archit. Compilation, Sep. 2015, pp. 113-124.

[7] D. Jeon et al., "A 23-mW face recognition processor with mostly-read 5T Memory in 40-nm CMOS," IEEE J. Solid-State Circuits, vol. 52, no. 6, pp. 1628-1642

[8] J. Zhang, Z. Wang, and N. Verma, (2017) "In-memory computation of a machine-learning classifier in a standard 6T SRAM array," IEEE J. Solid-State Circuits, vol. 52, no. 4, pp. 915924

[9] S. Jeloka, N. B. Akesh, D. Sylvester, and D. Blaauw, (2016) "A $28 \mathrm{~nm}$ configurable memory (TCAM/BCAM/SRAM) using push-rule 6T bit cell enabling logic-in-memory," IEEE $J$. Solid-State Circuits, vol. 51, no. 4, pp. 1009-1021

[10] A. T. Do, C. Yin, K. S. Yeo, and T. T.-H. Kim, (2013) "Design of a power-efficient CAM using automated background checking scheme for small match line swing," in Proc. IEEE Eur. Solid-State Circuits Conf. (ESSCIRC), Sep. pp. 209-212.

[11] M. Kang and N. R. Shanbhag, (2016) "In-memory computing architectures for sparse distributed memory," IEEE Trans. Biomed. Circuits Syst., vol. 10,no. 4, pp. 855-863

[12] S. Moriwaki, A. Kawasumi, T. Suzuki, T. Sakurai, and S. Miyano, (2011) "0.4 V SRAM with bit line swing suppression charge share hierarchical bit line scheme," in Proc. IEEE Custom Integr. Circuits Conf. (CICC), Sep. pp. 1-4.

[13] S. Lutkemeier, T. Jungeblut, H. K. O. Berge, S. Aunet, M. Porrmann, and U. Ruckert, (2013) "A $65 \mathrm{~nm} 32 \mathrm{~b}$ subthreshold processor with 9T multi-Vt SRAM and adaptive supply voltage control," IEEE J. Solid-State Circuits, vol. 48, no. 1, pp. 8-19

[14] A. T. Do, Z. C. Lee, B. Wang, I.-J. Chang, X. Liu, and T. T.-H. Kim, (2016) "0.2 V 8T SRAM with PVT-aware bitline sensing and column-based data randomization," IEEE J. Solid-State Circuits, vol. 51, no. 6, pp. 1487-1498

[15] N. Verma and A. P. Chandrakasan, (2008) "A $256 \mathrm{~kb} 65 \mathrm{~nm} 8 \mathrm{~T}$ subthreshold SRAM employing sense-amplifier redundancy," IEEE J. Solid-State Circuits, vol. 43, no. 1, pp. 141149

[16] Y. Sinangil and A. P. Chandrakasan, (2014) "A 128 kbit SRAM with an embedded energy monitoring circuit and sense-amplifier offset compensation using body biasing," IEEE $J$. Solid-State Circuits, vol. 49, no. 11, pp. 2730-2739 